\title{
Big Data Clustering Using Improvised Fuzzy C-Means Clustering
}

\author{
Venkat Rayala*, Satyanarayan Reddy Kalli
}

Research Centre, Department of CSE, Cambridge Institute of Technology, Affiliated to Visvesvaraya Technological University (VTU)-Belgaum, Bengaluru 560036, India

Corresponding Author Email: rayalavenkat534@gmail.com

https://doi.org/10.18280/ria.340604

Received: 24 October 2020

Accepted: 30 November 2020

\section{Keywords:}

Fuzzy C-Means (FCM), Convolutional

Neural Network (CNN), improvised Fuzzy

C-Means (IFCM)

\begin{abstract}
Clustering emerged as powerful mechanism to analyze the massive data generated by modern applications; the main aim of it is to categorize the data into clusters where objects are grouped into the particular category. However, there are various challenges while clustering the big data recently. Deep Learning has been powerful paradigm for big data analysis, this requires huge number of samples for training the model, which is time consuming and expensive. This can be avoided though fuzzy approach. In this research work, we design and develop an Improvised Fuzzy C-Means (IFCM)which comprises the encoder decoder Convolutional Neural Network (CNN) model and Fuzzy C-means (FCM) technique to enhance the clustering mechanism. Encoder decoder based CNN is used for learning feature and faster computation. In general, FCM, we introduce a function which measure the distance between the cluster center and instance which helps in achieving the better clustering and later we introduce Optimized Encoder Decoder (OED) CNN model for improvising the performance and for faster computation. Further in order to evaluate the proposed mechanism, three distinctive data types namely Modified National Institute of Standards and Technology (MNIST), fashion MNIST and United States Postal Service (USPS) are used, also evaluation is carried out by considering the performance metric like Accuracy, Adjusted Rand Index (ARI) and Normalized Mutual Information (NMI). Moreover, comparative analysis is carried out on each dataset and comparative analysis shows that IFCM outperforms the existing model.
\end{abstract}

\section{INTRODUCTION}

In recent times, enormous amount of data is being generated every day from various sources such as social media, satellites, sensors, mobile devices, computer simulations and business transaction. This data produces valuable information useful for business intelligence, forecasting, decision support, intensive data research. Walmart has nearly 2.5 petabytes and Facebook stores nearly 30 petabytes of data, such huge data is known as Big Data; mining such big data is necessary to extract the desired information [1-3]. In general data are classified into the three types i.e. Structured, Semi-structured and Unstructured. Major part of the data portion is unstructured data which cannot be handled through traditional method. Big data can be defined through three distinctive parameters volume, velocity and variety [4]. Velocity describes the speed at which the data is exchanged, captured, and generated. Variety of data refers to type of data i.e. data is not always available in the structured form. It explains the complexities.

Clustering is unsupervised; essential for analyzing the data, partitions data into various subsets in particular way that similar data is clustered $[5,6]$. Clustering structure can be defined through the below equation, let's considerC as the cluster set and $\mathrm{C}_{1}, \mathrm{C}_{2}$ etc be the clusters. Clustering is considered to be one of the machine learning mechanism.

$$
\mathrm{C}_{1} \cap \mathrm{C}_{2} \cap \mathrm{C}_{2} \ldots \ldots \mathrm{C}_{\mathrm{n}}=\varnothing
$$

Big Data Clustering can be described through two aspects single and multiple machine clustering. Single aims for consolidating the data objects in accordance with the specific parameter [7]; based on the partition which divides the dataset into the single partition through the distance for points classification based on their similarities. However, the drawback is, it requires the pre-defined parameter which is non-deterministic [8-10]. Figure 1 shows different types of Clustering. Euclidean distance computes the minimum distance observed among the available cluster and assigned points [11]. Existing clustering algorithm has advantage of simple implementation whereas drawback of this approach is that it fails miserably to deal with large amount of data.

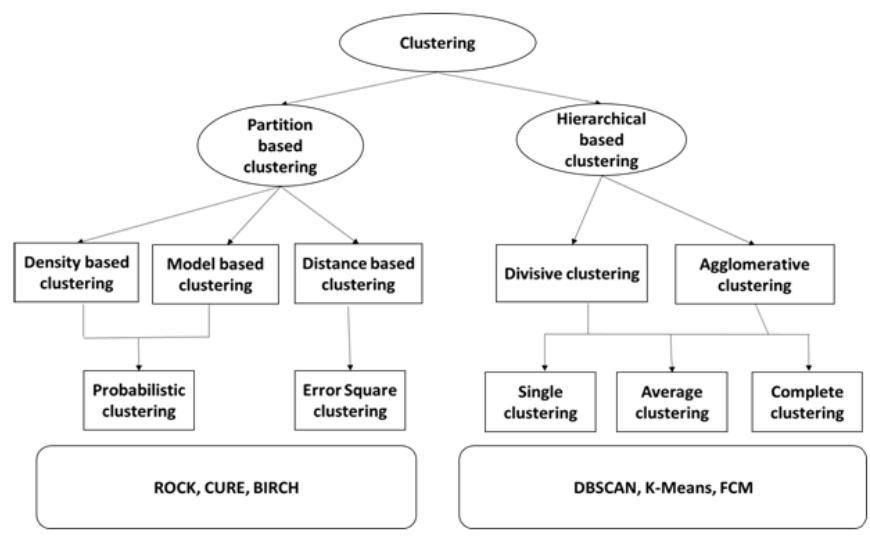

Figure 1. Types of clustering mechanism 


\subsection{Motivation and contribution of research work}

Clustering mechanism requires the absolute weights for weighted distance in accordance with requirement; the existing clustering approach uses assigned weights randomly. Moreover, fuzzy and neural network aims at exploiting the knowledge processing; through the survey it is observed that very few researchers have considered deep learning concept and none of them have considered encoder decoder based CNN. Motivated by this phenomenon we have developed IFCM which comprises encoder decoder CNN with FCM for better performance metrics.

In this work an IFCM is developed for efficient and high accuracy intended big data clustering.

IFCM comprises of distinctive framework namely FCM, FCM with function parameter and OED-CNN.

- FCM approach, we introduce a function which measure the distance between the Cluster Center (CC) and instance which helps in achieving the better clustering. Improvised FCM has Dual CNN for handling the big data in efficient manner.

- $\quad$ Later we introduce OED- CNN model for enhancing the performance metrics and faster computation.

IFCM is evaluated through considering the wellestablished data such as MNIST, fashion-MNIST, USPS; further comparative analysis is carried out with the existing model. IFCM performs better than several existing model.

\section{LITERATURE SURVEY}

In this section we review several existing methodology; at first VAT [12] discuss clustering through dissimilarity matrix to achieve the modified matrix such that various cluster are displayed as the dark block through diagonally which is used in the dark matter halos, however this works only for the large cluster data. Moshtaghi et al. [13] developed an approach clustering by anomaly detection; here dendograms were used for the visual representation and applied for several taxonomy applications [14]. Similarly, Wilbik et al. [15] proposed single linkage-based clustering for segmenting the time series based data to monitor the patient. The VAT commercial application was used for security [16], further it is observed that $\mathrm{K}$-means promises to cluster the data efficiently. The advantage of using K-means is its applicability and simplicity in several fields; as a batch based algorithm, it comes with various limitation as it has poor initialization. In recent years, deep learning has been one of the major research areas; a supervised learning task that has gained satisfactory results in big data clustering [17-20]; fails to deliver the result among the raw data and it affects the accuracy. Hence several rough based or fuzzy based approach is developed for handling the uncertainty in clustering. Deng et al. [17] developed a hierarchical approach which integrates the neural network and fuzzy logic for the robust clustering; here they minimize the vagueness. In literature [20], a fuzzy based CNN model was developed for the classification and clustering, in here at first CNN was applied to automate the feature extraction from given any input image and later FCM approach was used for clustering the data in defined feature space.

Rajesh et al. [21] Developed an approach based on neural network with rough set based to cluster the data. Set theory approach was used for extracting the feature and then produced as input for the Feed Forward (FF)-neural network to cluster data. This is succeeded in handling the data quiet well; however these are mainly supervised learning approach and requires huge data for training and this further causes the time consumption. Further semi-supervised clustering was introduced to handle the clustering and classification $[19,22$, 23]; Wu and Prasad [19] developed the restricted labeled data using the pseudo label. At first predicted label is used for clustering algorithm and pre-train neural network along with predicted labels. Predicted label helps in extracting the discriminating features; further ne-tune were introduced for adjusting the features from given pre-trained network for more beneficial to the clustering and classification. Tarvainen and Valpola [24] proposed semi-supervised learning named MT-model; MT-model averages the model weight for formatting the teacher model. MT-model was designed for the online learning and large dataset.

An efficient deep neural network was developed [25]; selfensemble was introduced to form the predicting the unknown label through network training the various epochs. Moreover, the above two mentioned performs great on the general dataset; but it fails on achieving the better accuracy on the noisy sample and uncertain dataset. Apart from this research work like the literatures [26, 27] focused on discussing advantage of FCM algorithm over the other clustering technique. Considering the above existing methodology, we observe that all this clustering mechanism faces problem of computational time, absolute clustering, and performance metrics. However, through the research gap analysis it was observed that FCM possesses a great potential in comparison with the other existing technique like $\mathrm{k}$-means or other traditional method. Most of the existing model follows hard clustering which categorizes the object into one category whereas FCM is soft clustering. However, FCM takes more computational time and fails in metrics, hence in the next section we design and develop improvised FCM which is based on the CNN mechanism.

\section{PROPOSED METHODOLOGY}

In this section, we develop Proposed Mechanism based on the CNN for enhancing the clustering mechanism. This is partitioned into various segments; at first, we learn about the general FCM and further we introduce a function parameter to compute the distance between the cluster center and instance. Later OED-CNN is introduced for improvisation in performance metrics. At last, both sub-mechanisms are integrated and presented as IFCM. In this section we discuss proposed model for big data representation. Let's define $\mathrm{Z} \in$ $\mathrm{T}^{\mathrm{K}_{1} \times \mathrm{K}_{2} \times \ldots \times \mathrm{K}_{1}}$ as $\mathrm{N}$-order multidimensional array with size of $\mathrm{K}_{1} \times \mathrm{K}_{2} \times \ldots \times \mathrm{K}_{\mathrm{P}}$; multi-dimensional array presents different big data types such as unstructured data, structured data and semi-structured data and the character strings which is stored in the rational database.

\subsection{Initialization}

In general clustering approaches, objects are assigned to the single cluster. Fuzzy concept allows objects to belong to more than single cluster. In this research work we modify the concept of FCM algorithm. 


\subsection{System model and general FCM algorithm}

FCM algorithm operates through assigning the membership for each data point to correspondent $\mathrm{CC}$ based on the data point and cluster distance; the main advantage of FCM is that it provides the outstanding result in case of overlapped data and also it assigns the data point to more than one cluster. However apart from computational time and accuracy it requires a greater number of iteration and Euclidean distance is used which measures the weight in unequal manner. Hence this can be reduced through encoder decoder based CNN.

Let us consider the dataset $\mathrm{Z}=\left\{\mathrm{z}_{1}, \mathrm{z}_{2}, \ldots, \mathrm{z}_{\mathrm{q}}\right\}$ with cluster set $X=\left\{x_{1}, x_{2}, \ldots . x_{p}\right\}$ and membership set $W=$

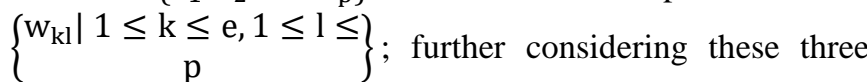

FCM can be formulated. In general, the idea of proposed mechanism is to integrate the IFCM with double neural network. Further we develop an optimized auto-encoder for training the for instance.

$$
\begin{gathered}
\min : \sum_{k=1}^{e} \sum_{l=1}^{p} w_{k l}^{o}\left\|z_{l}-x_{k}\right\|^{2} \\
\sum_{l=1}^{e} w_{k l}=1, w_{k l} \geq 0
\end{gathered}
$$

To avoid false clustering, we develop modified-FCM, Modified FCM in the below equation.

$$
\begin{aligned}
L_{o}(W, X)=\sum_{k=1}^{e} \eta_{i} & \sum_{k=1}^{p}\left(1-u_{k l}^{o}\right)^{o} \\
& +\sum_{k=1}^{e} \sum_{k=1}^{p} w_{k l}^{m}\left\|z_{l}-z_{i}\right\|^{2}
\end{aligned}
$$

Hence optimizing the equation helps in updating the membership matrix as well as cluster centers and given as:

$$
x_{k}=\sum_{l=1}^{p} w_{k l}^{o} z_{l} / \sum_{l=1}^{p} w_{k l}
$$

Membership matrix:

$$
w_{k l}=\left(1+\left(\frac{e_{k l}}{\eta_{k}}\right)^{-1 /(o-1)}\right)^{-1}
$$

In the above equation, $\mathrm{e}_{\mathrm{kl}}$ indicates the distance between the cluster and membership matrix. Table 1 below is the General FCM Algorithm.

Table 1. General FCM Algorithm

\begin{tabular}{c}
\hline Input: dataset, Max \\
\hline Output: optimized cluster member and membership vector \\
\hline Step1: Initialization of membership_matrix \\
\hline Step2: for $k=1$ to e do \\
\hline Step3: cluster center updation \\
\hline Step4: updating the fuzzy constant \\
\hline Step $5:$ for $=1$ to e do
\end{tabular}

\begin{tabular}{c}
\hline Step6: for l=1 to e do \\
\hline Step7: cluster center updation \\
\hline Step8: end for loop (step7) \\
\hline Step 9: end of for loop (step6) \\
\hline Step10: end of for loop (step 2$)$ \\
\hline
\end{tabular}

\subsection{Improvised Fuzzy C-Means approach}

\subsubsection{Function parameter}

In this section, the function parameter is introduced for computing the distance between the instance and $\mathrm{CC}$ for better clustering as FCM faces huge drawback due to the distance. In Improvised FCM each instance is considered as the multidimensional array for capturing the correlation over various modalities. Moreover, before deploying the FCM Optimized Encoder Decoder is applied for training the model, moreover to train the model Optimized Encoder Decoder is designed in the next section. Table 2 below is the modified FCM Algorithm

Table 2. Modified FCM Algorithm

\begin{tabular}{c}
\hline $\begin{array}{c}\text { Input: Dataset, } M, n, e \\
\text { Output: optimized cluster member and membership vec }\end{array}$ \\
\hline Step1: Initialization of membership matrix $V$ \\
\hline Step $2:$ for $k=1$ to $\mathbb{M}$ do \\
\hline Step $3:$ for $k=1$ to e do \\
\hline Step4: cluster center updation \\
$\eta_{k}=\sum_{l=1}^{o} w_{k l}^{l} f_{T D_{(k l)}} / \sum_{l=1}^{o} w_{k l}^{o}$ \\
\hline Step5: for $k=1$ to e do \\
\hline Step6: for $l=1$ to $p$ do \\
\hline Step7: $w_{k l}=\left(\left(1+\left(\frac{f_{T D}(k l)}{\eta_{i}}\right)^{-1 /(o-1)}\right)^{-1}\right)$ \\
\hline Step8: end of for loop(step6)
\end{tabular}

\subsubsection{Computational model}

Computational model utilizes the $\mathrm{CNN}$ as the basic module for pre-training the parameters which are time consuming and highly computational. Further we design the optimized version to reduce the time overhead and the computational without compromising the parameters. The optimized Neural Network takes input as $\mathrm{Z} \in \mathrm{T}^{\mathrm{K}_{1} \times \mathrm{K}_{2} \ldots . . \times \mathrm{K}_{\mathrm{P}}}$ and reconstruction of same is represented as $\mathrm{Z} \in$ $\mathrm{T}^{\mathrm{K}_{1} \times \mathrm{K}_{2} \ldots . . \times \mathrm{K}_{\mathrm{P}}}$

$$
\begin{aligned}
& \operatorname{hid}_{-} \operatorname{layer}_{l 1 \ldots . l_{P}}=\operatorname{enc}(\psi)\left(\sum_{k_{1} \ldots . k_{P}}^{K_{1} \ldots . . K_{P}} d_{l_{1} \ldots \ldots l_{P}}^{(1)}\right. \\
& \left.+Y_{\alpha k_{1} \ldots . k_{P}}^{(1)} Z_{k_{1 \ldots \ldots k_{P}}}\right) \\
& \text { out_layer }_{k_{1} \ldots k_{P}}=\operatorname{dec}(\psi)\left(\sum_{l_{1} \ldots . . l_{O}}^{L_{1} \ldots . L_{P}} d_{k_{1} \ldots \ldots . . k_{P}}^{(1)}\right. \\
& \left.+Y_{\beta l_{1} \ldots . . l_{O}}^{(1)} \text { hid_layer }{ }_{l_{1} \ldots . . . l_{O}}\right)
\end{aligned}
$$

In above equation, $\mathrm{K}_{1}$ indicates the number of dimension whereas $L_{1}$ indicates the hidden layer, enc is encoder and dec is decoder; further here we use sigmoid function in the encoding layer and decoding layer. Reconstruction objective 
is given through the below equation. Eq. (8) is objective of the current research, this is reconstruction objective.

$$
\begin{aligned}
& \mathrm{L}_{\mathrm{V} \text { encdec }}(\Psi) \\
& =\frac{1}{\mathrm{o}} \sum_{\mathrm{m}=1}^{\mathrm{o}}\left(\sum_{\mathrm{s}=1}^{\mathrm{K}_{1} \times \ldots . . \times \mathrm{K}_{\mathrm{O}}} \sum_{\mathrm{l}_{1}=1}^{\mathrm{L}_{1}} \ldots \sum_{\mathrm{l}_{\mathrm{O}}=1}^{\mathrm{L}_{\mathrm{P}}}\left(\mathrm{Y}_{\mathrm{sl}_{1} \ldots \ldots \mathrm{l}_{\mathrm{O}}}^{(2)}\right)^{2}\right. \\
& +\left(0.5\left(\text { out_layer }_{\mathrm{m}}-\mathrm{Z}_{\mathrm{m}}\right)\right)^{\mathrm{V}} \mathrm{I}\left(\text { out_layer }_{\mathrm{m}}\right. \\
& \left.\left.-\mathrm{Z}_{\mathrm{m}}\right)\right) \\
& \left.+0.5 \zeta\left(\sum_{\mathrm{r}=1}^{\mathrm{L}_{1} \times \ldots . . \times \mathrm{L}_{\mathrm{n}}} \sum_{\mathrm{k}_{1}=1}^{\mathrm{K}_{1}} \ldots \sum_{\mathrm{j}_{\mathrm{P}}=1}^{\mathrm{K}_{\mathrm{O}}} \mathrm{Y}_{\mathrm{rk}_{1} \ldots . . \mathrm{k}_{\mathrm{O}}}^{(1)}\right)^{2}\right]
\end{aligned}
$$

Further back propagation is used for training the parameter.

\subsubsection{Optimized Encoder Decoder CNN (OED-CNN)}

OED-CNN is designed to minimize the time and computational overhead without affecting the performance. Optimized ANN comprises two hidden layer. OED-CNN is same as the encoder decoder based CNN except here we introduce dual approach for better training of model. Figure 2 below is the OED-CNN Model.

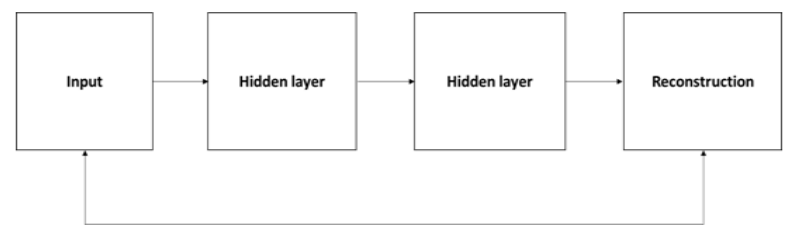

Figure 2. OED-CNN Model

OED-CNN takes an input as $\mathrm{Z} \in \mathrm{T}^{\mathrm{K}_{1} \times \mathrm{K}_{2} \ldots . . \times \mathrm{K}_{\mathrm{P}}}$; further operation at both the hidden layer is given through the respective equation.

$$
\begin{aligned}
& \text { hidden_layer } r_{1 l 1 \ldots . . l o} \\
& =\operatorname{enc}(\psi)\left(\sum_{k_{1} \ldots . . . K_{P}} Y_{\alpha j_{1} \ldots . . j_{P}}^{2(1)} Z_{j_{1} \ldots \ldots j_{P}}\right. \\
& \left.+d_{l 1 \ldots . . l o}^{(1)}\right)
\end{aligned}
$$

In above equation, hiddden_layer ${ }_{1}$ is the first layerand hiddeen_layer $r_{2} \in \mathrm{T}^{\mathrm{M}_{1} \times \mathrm{M}_{2} \ldots . . \times \mathrm{M}_{\mathrm{T}}}$.

$$
\begin{aligned}
& \text { hidden_layer } r_{2 m_{1} \ldots m_{T}} \\
& =\operatorname{enc}(\psi)\left(\sum_{l_{1} \ldots l_{P}}^{L_{1} \ldots L_{P}} Y_{\alpha l_{1} \ldots l_{P}}^{(1)} \text { hidden_layer }{ }_{l_{1} \ldots l_{P}}+d_{l 1 \ldots l o}^{(1)}\right.
\end{aligned}
$$

Similarly, hidden_layer ${ }_{1} \in \mathrm{T}^{\mathrm{L}_{1} \times \mathrm{L}_{2} \times \ldots . . \times \mathrm{L}_{\mathrm{N}}}$ indicates the second layer and Hidden_layer ${ }_{2} \in \mathrm{T}^{\mathrm{M}_{1} \times \mathrm{M}_{2} \ldots . . . \times \mathrm{M}_{\mathrm{O}}}$; in both equationsenc $(\psi)$ indicates the encoder. And the output is given through $\mathrm{Y}$ and represented in the below equation; here $\operatorname{dec}(\psi)$ indicates the decoder.

$$
\begin{aligned}
& \text { output_layer }_{k 1 \ldots k o} \\
& =\operatorname{dec}(\psi)\left(\sum_{l_{1} \ldots l_{O}}^{M_{1} \ldots M_{T}} d_{k 1 \ldots k O}^{(2)}\right. \\
& +Y_{\beta k_{1} \ldots k_{O}}^{(1)} \text { hidden_layer }{ }_{2 m_{1} \ldots m_{T}} \text { ) }
\end{aligned}
$$

Moreover, in the optimized ANN training model, Rectifier unit is used in the encoder function as the activation function and it is given as:

$$
h^{\prime}(z)= \begin{cases}1 & z>0 \\ 0 & z \leq 0\end{cases}
$$

Further parameters are trained and reconstruction function is given as:

$$
L_{A T A E}(\psi)=0.5(a-z)^{T} I(a-z)
$$

In the above equation, $\mathrm{z}$ and $\mathrm{a}$ are the vector; Idenotes the coefficient. Further the reconstruction function on the given $\mathrm{m}$ training sample is given as:

$$
\begin{aligned}
L_{G R F}(\psi)= & {\left[0.5 \sum_{k=1}^{m} 0.5 \zeta\left(Y^{(1) 2}+Y^{(2) 2}+Y^{(3) 2}\right)^{2}\right.} \\
& \left.+0.5(a-z)^{T} I(a-z)\right]
\end{aligned}
$$

Later, back propagation is applied for computing the $\delta \mathrm{Y}$.

$$
\mathrm{Y}=Y-\varphi\left(\frac{1}{o} \sum_{k=1}^{o} \varphi Y+\delta Y_{k}\right)
$$

Similarly back propagation is applied for computing $\Delta \mathrm{d}$. Eq. (16) is the back propagation is applied for computing $\Delta \mathrm{d}$.

$$
\Delta \mathrm{d}=\mathrm{d}-\varphi\left(\frac{1}{\mathrm{o}} \sum_{\mathrm{k}=1}^{\mathrm{o}} \delta \mathrm{d}_{\mathrm{k}}\right)
$$

In the above equation $\varphi$ indicates the rate of learning. Moreover in order to compute the derivative in case of each sample, forward propagation is applied for input and output value computation. Eq. (17) is Forward Propagation for input, Eq. (18) is the Forward Propagation of output.

$$
\begin{gathered}
\tau_{k}^{(4)}=\left(\sum_{l=1}^{K_{1} \times \ldots \ldots \times K_{O}} i_{k l}\left(z_{k}^{(3)}-a_{k}\right)\right) \cdot h^{\prime}\left(b_{k}^{(4)}\right) \\
\tau_{m_{1} m_{2} \ldots m_{T}}^{(3)}=\left(\sum_{l=1}^{K_{1} \times \ldots \ldots \times K_{O}} Y_{k l_{1} \ldots m_{T}}^{(3)} \cdot \tau_{k}^{(4)}\right) \cdot h^{\prime}\left(b_{k}^{(4)}\right)
\end{gathered}
$$

In above equation, $\tau_{1_{1}, l_{2} \ldots l_{T}}^{(4)}$ and $\tau_{11,12 \ldots, 10}^{(3)}$ are input and output values; error is computed for each neuron through below equation.

$$
\tau_{m_{1} m_{2} \ldots m_{T}}^{(2)}=\frac{\partial L_{\text {Genecdec }}^{(4)}(\varphi)}{\partial b_{l_{1} \ldots l_{P}}^{(3)}}
$$

Further partial derivative of $\frac{\partial \mathrm{b}^{(4)}}{\partial \mathrm{Y}^{(\mathrm{n})}}$ is computed by considering $\mathrm{n}=1,2$ and 3 .

$$
c_{m_{1} m_{2} \ldots m_{T}}^{(3)}=\frac{\partial b_{k_{1} \ldots k_{O}}^{(4)}}{\partial Y_{l_{1} \ldots l_{S}}^{(3)}}
$$




$$
\begin{gathered}
c_{m_{1} m_{2} \ldots m_{T}}^{(2)}=\frac{\partial b_{k_{1} \ldots k_{O}}^{(3)}}{\partial Y_{l_{1} \ldots l_{S}}^{(2)}} \\
c_{m_{1} m_{2} \ldots m_{T}}^{(1)}=\frac{\partial b_{j_{1} \ldots j_{O}}^{(2)}}{\partial Y_{k_{1} \ldots k_{R}}^{(1)}}
\end{gathered}
$$

Considering the chain rule we compute the derivatives of $\delta \mathrm{Y}$ and $\Delta \mathrm{d}$.

$$
\begin{gathered}
\Delta Y^{(m)}=\frac{\partial L_{G e n c d e c}(\psi)}{\partial b^{(n+1)}} \cdot \frac{\partial L^{(n+1)}}{\partial Y^{(n+1)}} \\
\Delta d^{(m)}=\varsigma^{(m+1)}
\end{gathered}
$$

Further Table 3 provides the whole process of improvised FCM with OED-CNN model.

Table 3. Improvised FCM with OED-CNN model

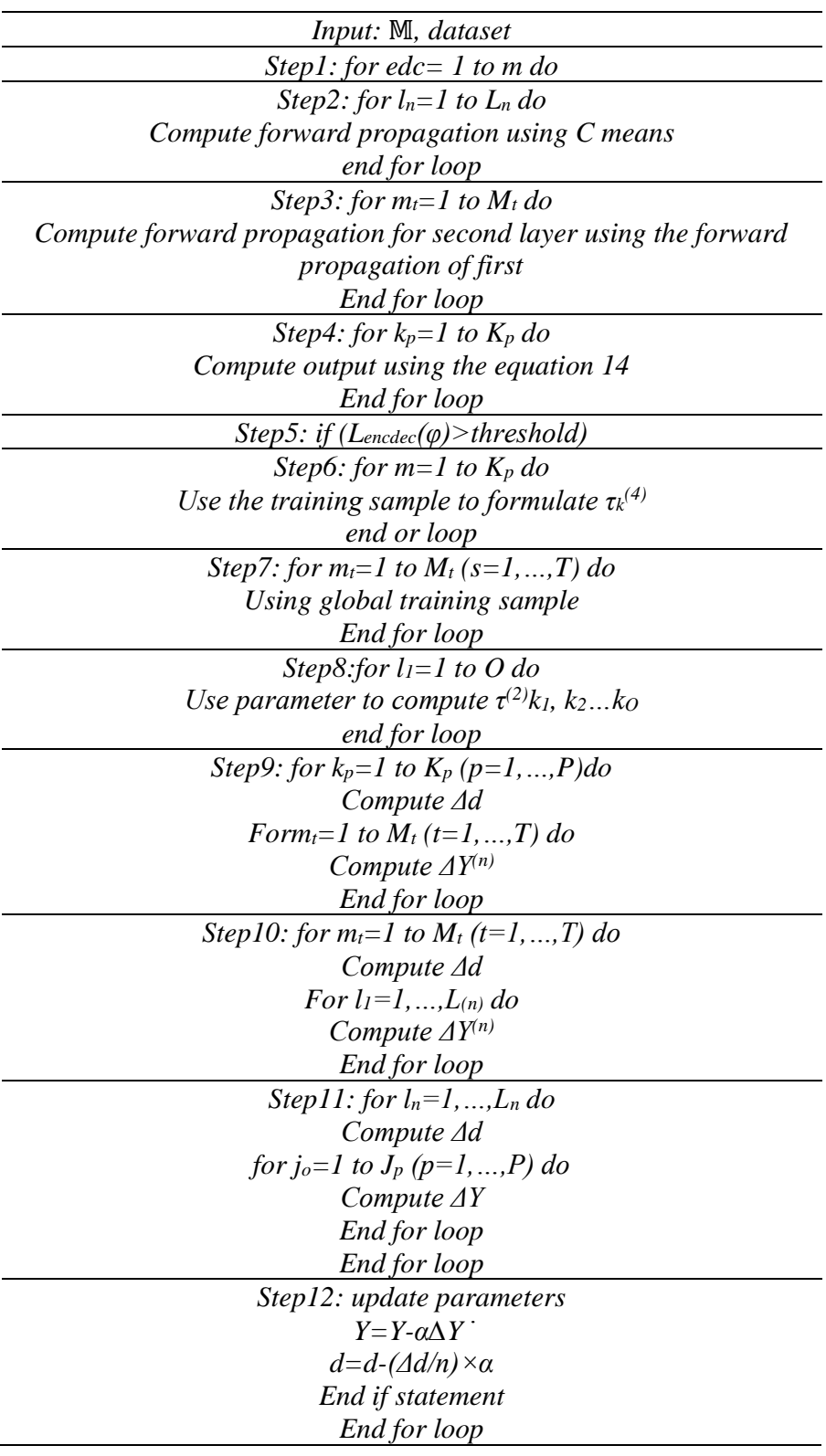

\section{PERFORMANCE EVALUATION}

In this section, the proposed mechanism is evaluated on real dataset for clustering; further comparative analysis is carried out. In order to evaluate the mechanism ideal system configuration of i7 processor packed with 2GB Nvidia graphics and 8GB RAM; further python is used as the programming language along with various machine learning libraries.

\subsection{Dataset details}

In this section, we provide a detailed description regarding the dataset; moreover three distinctive world dataset as MNIST, Fashion-MNIST and USPS; these dataset is considered for clustering. Fashion-MNIST is one of the popular fashion clothing dataset.

\subsection{Comparison algorithm}

Fuzzy C-Means: This uses the membership matrix and update rule for clustering.

K-means: Here data can belong to one particular cluster.

SEC: This is mainly based on the manifold learning.

MBKM: This algorithm is improvisation of K-means algorithm where mini-batch is used for minimizing the computational complexity.

DEC: This algorithm is mainly based on the deep learning, further this clustering model is based on the particular designed distribution and abandons the decoder part.

IDEC: This is one of the deep clustering models; further this clustering model is based on the particular designed distribution and uses the reconstruction mechanism for regularizing the auto encoder.

\subsection{Performance metrics}

\subsubsection{Normalized Mutual Information (NMI)}

In general, mutual information is defined as the measure of mutual dependence between two variables. NMI aka normalization of mutual information lies between 0 to 1,0 indicates no mutual information and 1 indicates the perfect correlation. Higher NMI value indicates the better clustering model.

$$
N M I=(H(E)+H(A))(H(E, A))^{-1}
$$

\subsubsection{Adjusted Rand Index (ARI)}

Rand Index is nothing but measure of similarity between two distinctive data clustering, Rand Index has value of range between 0 and 1, 0 indicates that two distinctive data clustering at any point and 1 indicates that data clustering is absolute. Higher value of ARI indicates the higher efficiency of model.

$$
\begin{aligned}
& \text { Average Rand index } \\
& \qquad \begin{aligned}
& (\text { Rand_Index } \\
& - \text { true negative }) \\
& /(\max (\text { Rand_Index }) \\
& -E(\text { Rand_Index }))^{-1}
\end{aligned}
\end{aligned}
$$

IFCM provides the better and faster clustering accuracy. 


\subsubsection{Accuracy}

\section{Clustering_accuracy}

$$
=P\left(\sum_{k=1}^{P} 1\left(A_{k}=\max \left(d_{k}\right)\right)\right)^{-1}
$$

In the above equation, $d_{k}$ indicates the clustering assignment.

4.3.4 Modified National Institute of Standards and Technology (MNIST) dataset

In this section, a comparative analysis of various method based on the three discussed metric is carried out. In here, it is observed that FCM achieves the very less accuracy of $54.68 \%$, whereas other method like K-means and MBKM fails miserably with accuracy of $53.48 \%$ and $54.43 \%$. Further the other improvised methodology promises for better accuracy with $97.71 \%$ existing model achieving $91.45 \%$. Similarly, in terms of ARI, FCM and K-means remains on the lower side with ARI value of $36.96 \%$ and $36.67 \%$; other method like IDEC, DEC shows the marginal improvement with $88.01 \%$ and $86.53 \%$ respectively. Moreover, existing model achieves ARI value of $86.26 \%$; however in comparison with that Improvised model achieves massive ARI of $93.87 \%$. Furthermore, considering the NMI as metrics method like FCM and K-means achieves $48.16 \%$ and $49.99 \%$ respectively; improvising this existing model achieves NMI value of $90.74 \%$. In comparison with this entire model our model achieves $95.02 \%$. Table 4 below is the performance metric comparison on MNIST dataset and accuracy graph is shown in the Figure 3.

Table 4. Performance metric comparison on MNIST dataset

\begin{tabular}{cccc}
\hline Clustering Methodologies & Accuracy & ARI & NMI \\
\hline Fuzzy C-Means & 54.68 & 36.96 & 48.16 \\
SEC[28] & 62.73 & 48.59 & 60.38 \\
K-means & 53.48 & 36.67 & 49.99 \\
MBKM[29] & 54.43 & 36.85 & 44.82 \\
IDEC[30] & 88.01 & 83.25 & 86.38 \\
DEC[31] & 86.53 & 80.29 & 83.69 \\
GrDFCM & 90.24 & 84.97 & 88.67 \\
DFCM & 88.17 & 83.37 & 86.54 \\
DNFCS & 88.26 & 83.44 & 86.65 \\
GrDNFCS[32] & 91.45 & 86.26 & 90.74 \\
Improvised_FCM & 97.71 & 93.874 & 95.024 \\
\hline
\end{tabular}

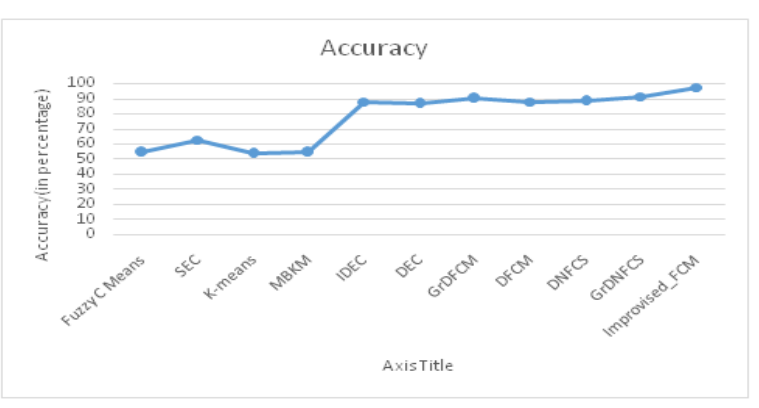

Figure 3. Comparison of various existing model on MNIST dataset

\subsubsection{United States Postal Service (USPS)}

Further evaluation of improvised FCM is carried out considering the comparison metric as accuracy, ARI and NMI on USPS dataset; Table 5 presents the comparison. In here existing method like fuzzy C-means achieves decent accuracy of $66.34 \%$ and $\mathrm{K}$ means achieves $66.79 \%$. Other existing method like DFCM and DNFCS shows some promising result with accuracy of $75.36 \%$ and $75.8 \%$ respectively. Moreover existing model i.e. GRDNFCS achieves $76.52 \%$ whereas IFCM achieves massive accuracy of $95.12 \%$. Further considering the ARI metric FCM and Kmeans achieves ARI of $53.93 \%$ and $54.5 \%$; other model like DFCM and DNFCS shows the decent improvisation with ARI of 68.15 and $68.77 \%$ respectively. In comparison with all these existing mechanism, Improvised FCM achieves $85.01 \%$. At last, considering the NMI metric, Fuzzy C-Means achieves NMI of $68 \%$ and $64.88 \%$; further DFCM and DNFCS shows promising with NMI of $76.36 \%$ and $76.96 \%$. Moreover, in comparison with all these method and existing model, proposed model achieves highest NMI of $89.01 \%$. Figure 4 shows the comparison graph in terms of accuracy.

Table 5. Performance metric comparison on USPS dataset

\begin{tabular}{cccc}
\hline Clustering Methodologies & Accuracy & ARI & NMI \\
\hline Fuzzy C-Means & 66.34 & 53.93 & 62 \\
SEC & 65.19 & 49.36 & 64.88 \\
K-means & 66.79 & 54.5 & 62.56 \\
MBKM & 62.87 & 51.05 & 59.93 \\
IDEC & 75.13 & 67.91 & 75.95 \\
DEC & 72.78 & 66.22 & 73.52 \\
GrDFCM & 76.03 & 68.83 & 77.25 \\
DFCM & 75.36 & 68.15 & 76.36 \\
DNFCS & 75.8 & 68.77 & 76.96 \\
GrDNFCS & 76.52 & 69.03 & 77.61 \\
Improvised_FCM & 95.12 & 85.01 & 89.01 \\
\hline
\end{tabular}

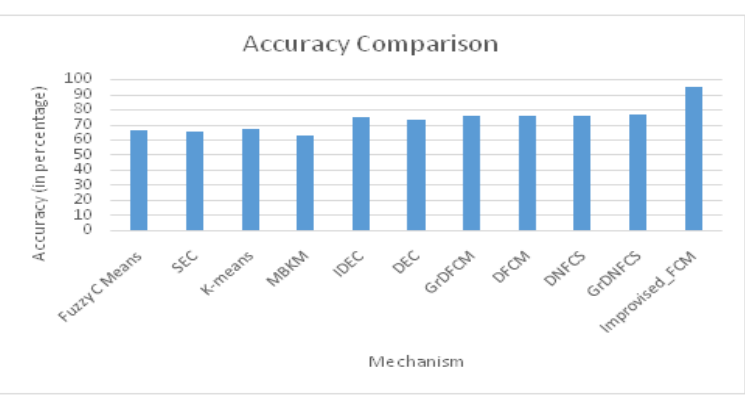

Figure 4. Comparison of various existing model on USPS dataset

\subsubsection{Fashion MNIST}

In this sub-section comparative analysis is carried out on the Fashion MNIST dataset; it is one of the most complicated dataset. Table 6 shows the comparison of various existing mechanism with proposed model in terms of accuracy, ARI and NMI. Moreover, Basic Fuzzy C-means achieves accuracy of $52.91 \%$ and K-means achieves accuracy of $51.07 \%$. However other method like IDEC, DEC, DFCM achieves better accuracy but it stays on lower side; furthermore improvised FCM achieves decent accuracy of $66.2 \%$ in comparison with existing model of $63.51 \%$. Similarly considering ARI as comparison metric, it is observed that Fuzzy C-means achieve ARI value of $36.44 \%$ and K-means achieves ARI value of $36.39 \%$; other existing model gives decent improvisation with DFCM achieving $48.65 \%$ and existing model achieving 50.28\%. Besides, in comparison with other existing model, Improvised FCM achieves decent ARI value of $54.19 \%$. Finally, NMI is considered as the comparison metric, where Fuzzy C-means 
achieves $51.59 \%$ and $\mathrm{K}$ means achieves $51.64 \%$. Moreover, existing model achieves $66.09 \%$ whereas improvised FCM achieves $67.35 \%$

Figure 5 below is comparison of various existing model on Fashion MNIST dataset.

Table 6. Performance Metric Comparison on Fasion MNIST dataset

\begin{tabular}{cccc}
\hline Clustering Methodologies & Accuracy & ARI & NMI \\
\hline Fuzzy C-Means & 52.91 & 36.44 & 51.59 \\
SEC & 54.24 & 38.44 & 55.8 \\
K-means & 51.07 & 36.39 & 51.64 \\
MBKM & 50 & 34.5 & 50.03 \\
IDEC & 57.64 & 44.09 & 60.13 \\
DEC & 57.81 & 45.71 & 62.83 \\
GrDFCM & 62.78 & 50.14 & 65.78 \\
DFCM & 62.29 & 48.65 & 64.54 \\
DNFCS & 62.5 & 49.91 & 65.67 \\
GrDNFCS & 63.51 & 50.28 & 66.09 \\
Improvised_FCM & 66.2 & 54.19 & 67.35 \\
\hline
\end{tabular}

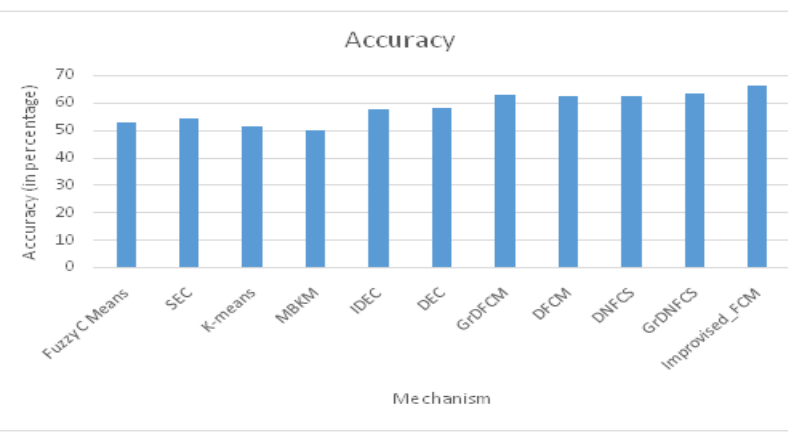

Figure 5. Comparison of various existing model on Fashion MNIST dataset

\section{CONCLUSION}

IFCM comprises the general FCM with additional function parameter for computing the distance between instance and $\mathrm{CC}$; Further we introduce OED-CNN to enhance the performance metrics. Moreover optimized encoder decoder CNN helps in training the model in efficient and faster way; combined with fuzzy C-Means, IFCM possesses fine clustering model. Further to evaluate IFCM, three established machine learning datasets are considered i.e. MNIST, Fashion-MNIST and USPS. Also, detailed comparative analysis is carried out considering performance metric as accuracy, normalized mutual index and adjusted rand index; in each of these metric IFCM excels in comparison with various state-of-art techniques like FCM and K-means. In machine learning area, clustering is considered as novice mechanism for data analysis; although IFCM possesses great clustering mechanism with marginally growth in comparison with other exiting models. There are several other areas which need to be focused for real time data clustering.

\section{REFERENCES}

[1] Chen, C.L.P., Zhang, C.Y. (2014). Data-intensive applications, challenges, techniques and technologies: A survey on Big Data. Inf. Sci. (Ny)., 275: 314-347. https://doi.org/10.1016/j.ins.2014.01.015
[2] Wang, X.K., Yang, L.T., Liu, H.Z., Deen, M.J. (2017). A big data-as-a-service framework: State-of-the-art and perspectives. IEEE Trans. Big Data, 4(3): 325-340. https://doi.org/10.1109/TBDATA.2017.2757942

[3] Elkano, M., Sanz, J.A.A., Barrenechea, E., Bustince, H., Galar, M. (2019). CFM-BD: A distributed rule induction algorithm for building Compact fuzzy models in big data classification problems. IEEE Trans. Fuzzy Syst., vol. https://doi.org/10.1109/TFUZZ.2019.2900856

[4] Kumar, D., Bezdek, J.C., Palaniswami, M., Rajasegarar, S., Leckie, C., Havens, T.C. (2016). A hybrid approach to clustering in big data. IEEE Transactions on Cybernetics, 46(10): 2372-2385. https://doi.org/10.1109/TCYB.2015.247741

[5] Deng, Y., Ren, Z.Q., Kong, Y.Y., Bao, F., Dai, Q.H. (2017). A hierarchical fused fuzzy deep neural network for data classification. IEEE Trans. Fuzzy Syst., 25(4): 1006-1012. https://doi.org/10.1109/TFUZZ.2016.2574915

[6] Blum, A.L., Langley, P. (1997). Selection of relevant features and examples in machine learning. Artif. Intell., 97(1-2): 245-271. https://doi.org/10.1016/S00043702(97)00063-5

[7] Han, J., Kamber, M., Pei, J. (2011). Data Mining: Concepts and Techniques. Morgan Kaufmann Publishers.

[8] Rokach, L. (2005). Clustering Methods. Data Mining and Knowledge Discovery Handbook. Springer, pp. 331-352.

[9] Saxena, A., Pal, N.R., Vora, M. (2010). Evolutionary methods for unsupervised feature selection using Sammon ${ }^{e c}$ stress function. Fuzzy Information and Engineering, 2: 229-247. https://doi.org/10.1007/s12543-010-0047-4

[10] Jain, A.K. (2008). Data clustering: 50 years beyond kmeans. Pattern Recognition Letters, 31(8): 651-666. https://doi.org/10.1016/j.patrec.2009.09.011

[11] Estivill-Castro, V., Yang, J.H. (2000). Fast and robust general purpose clustering algorithms. In: Mizoguchi R., Slaney J. (eds) PRICAI 2000 Topics in Artificial Intelligence. PRICAI 2000. Lecture Notes in Computer Science, vol 1886. Springer, Berlin, Heidelberg. https://doi.org/10.1007/3-540-44533-1 24

[12] Bezdek, J.C., Hathaway, R.J. (2002). VAT: A tool for visual assessment of (cluster) tendency.In Proc. Int. Joint Conf. Neural Netw. (IJCNN), Honolulu, HI, USA, pp. $2225-2230$ https://doi.org/10.1109/IJCNN.2002.1007487

[13] Moshtaghi, M., Havens, T.C., Bezdek, J.C., Park, L., Leckie, C., Rajasegarar, S., Keller, J.M., Palaniswami, M. (2011). Clustering ellipses for anomaly detection. Pattern Recognit., 54-69. https://doi.org/10.1016/j.patcog.2010.07.024

[14] Sneath, P.H.A., Sokal, R.R. (1973). Numerical Taxonomy-The Principles and Practice of Numerical Classification. San Francisco, CA, USA: W.H. Freeman\& Co.

[15] Wilbik, A., Keller, J.M., Bezdek, J.C. (2013). Linguistic prototypes for data from eldercare residents. IEEE Trans. Fuzzy Syst., 22(1): 110-123. https://doi.org/10.1109/TFUZZ.2013.2249517

[16] Zhang, D., Ramamohanarao, K., Versteeg, S., Zhang, R. (2009). RoleVAT: Visual assessment of practical need 
for role based access control. in Proc. Conf. Comput. Security Appl., Honolulu, HI, USA, pp. 13-22. https://doi.org/10.1109/ACSAC.2009.11

[17] Deng, Y., Ren, Z.Q., Kong, Y.Y., Bao, F., Dai, Q.H. (2017). A hierarchical fused fuzzy deep neural network for data classification. IEEE Trans. Fuzzy Syst., 25(4): 1006-1012. https://doi.org/10.1109/TFUZZ.2016.2574915

[18] Riaz, S., Arshad, A., Jiao, L.C. (2018). Fuzzy rough Cmean based unsupervised $\mathrm{CNN}$ clustering for largescale image data. Appl. Sci., 8(10): 1-20. https://doi.org/10.3390/app8101869

[19] Wu, H., Prasad, S. (2018). Semi-supervised deep learning using pseudo labels for hyperspectral image classification. IEEE Trans. Image Process., 27(3): 12591270. https://doi.org/10.1109/TIP.2017.2772836

[20] Yeganejou, M., Dick, S. (2018). Classification via deep fuzzy c-means clustering. 2018 IEEE International Conference on Fuzzy Systems (FUZZ-IEEE), Rio de Janeiro, pp. 1-6. https://doi.org/10.1109/FUZZIEEE.2018.8491461

[21] Rajesh, T., Malar, R.S.M. (2013). Rough set theory and feed forward neural network based brain tumor detection in magnetic resonance images. International Conference on Advanced Nanomaterials \& Emerging Engineering Technologies, Chennai, pp. 240-244. https://doi.org/10.1109/ICANMEET.2013.6609287

[22] Kuznietsov, Y., Stuckler, J., Leibe, B. (2017). Semisupervised deep learning for monocular depth map prediction.In Proc. IEEE Conf. Comput. Vis. Pattern Recognit., pp. 6647-6655.

[23] Zhou, S.S., Chen, Q.C., Wang, X.L. (2014). Fuzzy deep belief networks for semi-supervised sentiment classification. Neurocomputing, 131: 312-322. https://doi.org/10.1016/j.neucom.2013.10.011

[24] Tarvainen, A., Valpola, H. (2017). Mean teachers are better role models: Weight-averaged consistency targets improve semi-supervised deep learning results. In Proc. Adv. Neural Inf. Process. Syst., pp. 1195-1204.

[25] Laine, S., Aila, R. (2016). Temporal ensembling for semi-supervised learning. [Online]. Available: https://arxiv.org/abs/1610.02242

[26] Venkat, R., Reddy, K.S. (2019). Dealing big data using fuzzy c-means (FCM) clustering and optimizing with gravitational search algorithm (GSA). 2019 3rd International Conference on Trends in Electronics and Informatics (ICOEI), Tirunelveli, India, pp. 465-467. https://doi.org/10.1109/ICOEI.2019.8862673

[27] Venkat, R., Reddy, K.S. (2020). Clustering of huge data with fuzzy c-means and applying gravitational search algorithm for optimization. International Journal of Recent Technology and Engineering (IJRTE), 8(5): 3206-3209. https://doi.org/10.35940/ijrte.D9130.018520

[28] Nie, F.P., Xu, D., Tsang, I.W., Zhang, C.S. (2009). Spectral embedded clustering. IJCAI, pp. 1181-1186.

[29] Sculley, D. (2010). Web-scale k-means clustering. Proceedings of the 19th international conference on World wide web. ACM, pp. 1177-1178. https://doi.org/10.1145/1772690.1772862

[30] Xie, J.Y., Girshick, R., Farhadi, A. (2016). Unsupervised deep embedding for clustering analysis. International conference on machine learning, 48: 478487.

[31] Guo, X.F., Gao, L., Liu, X.W., Yin, J.P. (2017). Improved deep embedded clustering with local structure preservation. International Joint Conference on Artificial Intelligence (IJCAI-17), pp. 1753-1759. https://doi.org/10.24963/ijcai.2017/243

[32] Feng, Q.F., Chen, L., Chen, C.L.P., Guo, L. (2020). Deep fuzzy clustering-A representation learning approach. IEEE Transactions on Fuzzy Systems, 28(7): 1420-1433. https://doi.org/10.1109/TFUZZ.2020.2966173 